\title{
Nueva Licenciatura en Ingeniería en Nanotecnología en Ensenada
}

\section{LAURA Viana CaSTRILlón*}

Q uiero hacer de su conocimiento que se encuentra en proceso el desarrollo de una nueva licenciatura de la UNAM que será denominada "Ingeniería en Nanotecnología". Dicha licenciatura se impartirá en el recién creado Centro de Nanociencias y Nanotecnología de la UNAM (CNyN), que se ubica en Ensenada, Baja California y esperamos poder recibir a la primera generación de estudiantes durante el segundo semestre del 2009.

El cnyn tiene como objetivo realizar investigación de vanguardia en Física Teórica, Nanoestructuras, Materiales Avanzados, Fisicoquímica de Nanomateriales y Nanocatálisis. Otro de sus objetivos es formar recursos humanos de alta calidad en las áreas, disciplinas y técnicas relacionadas con estos temas. El cnyn cuenta con personal calificado en los aspectos físicos y químicos de las nanociencias y con infraestructura de primer nivel, por lo que ofrece muchas oportunidades para capacitar a estudiantes en nuevas tecnologías. Su personal cuenta con experiencia docente debido a que participa en tres programas de maestría y tres de doctorado, todos inscritos en el Programa Nacional de Posgrados de Calidad. A la fecha se han graduado 74 doctores y el $80 \%$ de sus doctores egresados residentes en México son miembros del SNI.

El objetivo de este proyecto es crear una licenciatura de excelencia a nivel nacional. Se buscará formar profesionales que cuenten con una sólida preparación interdisciplinaria de nivel básico, con conocimientos de técnicas de análisis y manipulación de materiales, con habilidades de comunicación, liderazgo y de trabajo en equipo, con entrenamiento en la solución de problemas prácticos y con los conocimientos y conciencia social que les permitan ubicarse en su entorno con eficiencia, responsabilidad y con espíritu de servicio. Por lo anterior, el plan de estudios incluye actividades de reto intelectual, formativas, de análisis, discusión, trabajo en equipo, para que los estudiantes aprendan a pensar, argumentar, reflexionar y decidir con responsabilidad, siendo capaces de hacerlo de forma autodidacta.

Aunque pudiera parecer que una licenciatura de Ingeniería en Nanotecnología es una carrera de alto grado de especialización, es mucho más que esto, ya que se encuentra entre la frontera de las ciencias y las ingenierías y permite que el alumno adquiera una amplia cultura científico-tecnológica y desarrolle una serie de herramientas que le permitirá ser un profesional competitivo en una amplia gama de trabajos o proseguir con estudios de posgrado, teniendo una base formativa sólida, general e interdisciplinaria, además de tener preparación de frontera en el área de las nanociencias y la nanotecnología.

La licenciatura está dirigida a estudiantes con alta capacidad analítica que gustan afrontar retos, que tienen gran imaginación, curiosidad y creatividad, que son independientes y muestran dedicación, constancia y disciplina en el trabajo, con inclinación hacia las ciencias físicas, biológicas y químicas, con interés en el ejercicio de las matemáticas y conocimientos básicos del idioma Inglés. Se espera que los alumnos dediquen 40 horas a la semana a los cursos, estudios y tareas, y que tengan disponibilidad para asistir a clases en los turnos matutino y vespertino y para prepararse en el estudio del idioma

\footnotetext{
* Coordinadora del Proyecto Ingeniería en Nanotecnología del Centro de Nanociencias y Nanotecnología de la UNAM. laura@cnyn. unam.mx; www.cnyn.unam.mx
} 
Inglés. En este sentido, se tiene contemplado poner a disposición de los alumnos, durante los primeros semestres, un laboratorio para el aprendizaje del idioma. Posteriormente, en los últimos semestres, el plan de estudios incluirá asignaturas de producción oral y escrita en Inglés técnico. Todo esto, bajo la supervisión del Centro de Enseñanza de Lenguas Extranjeras de la UNAM.

El egresado tendrá independencia de pensamiento y creatividad, rigurosidad en la deducción aunada a un alto nivel de manejo de las matemáticas y expresión clara de forma verbal y escrita en Inglés y en Español. Podrá participar en grupos interdisciplinarios que desarrollan labores de difusión científica. Tendrá entrenamiento para resolver problemas de la vida diaria utilizando sus conocimientos científicos. Contará con conocimientos técnicos para aplicar técnicas de preparación, síntesis, caracterización, diseño y aplicación de materiales. Tendrá hábitos de trabajo apropiados para ambientes de laboratorio. El plan incluye tres ejes de asignaturas optativas en Biotecnología, Ingeniería Ambiental y Nanoestructuras, todas con énfasis especial en las aplicaciones de la nanotecnología en estos campos.

El ingreso a la Licenciatura de Ingeniería en Nanotecnología de la UNAM será indirecto. Esto significa que para ingresar, los aspirantes deberán: i) haber concluido el ciclo de estudios inmediato anterior (bachillerato) con un promedio mínimo de siete o su equivalente; ii) ser aceptado al ciclo de licenciatura de la UNAM a cualquier carrera de las áreas de Ciencias Físico-Matemáticas, Ciencias Químico-Biológicas o de las Ingenierías, lo cual puede lograrse mediante pase reglamentado o como resultado del concurso de selección; iii) solicitar por escrito su registro en el Proceso de Selección de los Alumnos de Ingeniería en Nanotecnología, o hacer el registro por Internet en la página del cNyn; iv) ser seleccionado por la Comisión de Ingreso a la Licenciatura de Ingeniería en Nanotecnología con base en el Proceso de Ingreso establecido por dicha Comisión.

Creemos que esta licenciatura, enriquecerá la oferta académica del país y que representa una magnífica oportunidad para estudiantes con inquietudes científicas y con mentalidad práctica que tengan muchos deseos de prepararse en los temas técnicos y científicos de frontera, en un ambiente de investigación.

Ensenada, B.C., a 2 de septiembre de 2008. 